\title{
Improving Sentiment Analysis of Arabic tweets
}

\author{
Abdulrahman Alruban ${ }^{1}$, Muhammed Abduallah ${ }^{1}$, Gueltoum Bendiab ${ }^{1}$, Stavros \\ Shiaeles $^{2}$, and Marco Palomino ${ }^{1}$ \\ 1 CSCAN, University of Plymouth, PL4 8AA, Plymouth, UK \\ a.alruban@mu.edu, muhammed.abduallah@postgrad.plymouth.ac.uk, \\ bendiab.kelthoum@umc.edu.dz, marco.palomino@plymouth.ac.uk \\ 2 University of Portsmouth, Portsmouth, UK \\ sshiaeles@ieee.org
}

\begin{abstract}
Twitter, is a rich data collection source of feelings, ideas and opinions. As its popularity grows it attracts more research interest and specifically in sentiment analysis. The aim of this research was to build a system able to bridge the gap in research with regards to Twitter Arabic users and sentiment analysis by providing a novel method to classify Arabic Tweets with different Arabic dialects and emotions, as positive, negative or natural. The study provides quantitative analysis to gain in-depth understanding of the phenomenon under investigation and the findings of the study show that the designed system was successful in detecting negative, positive and neutral tweets.
\end{abstract}

Keywords: Sentiment Analysis. Security Arabic language - Twitter · Lexicon· Bots

\section{Introduction}

Nowadays, social networks offer powerful platforms where millions of people can easily share their thoughts, feelings and opinions about a wide variety of topics [17].A recent report by twitter Inc affirm that there are over 326 million of monthly active users involved with Tweeter in 2019, where $46 \%$ of them are on the platform daily, sending over 6,000 tweets every second, which corresponds to over 350,000 tweets per minute and 500 million tweets per day [13]. From his side, Facebook boasts 2.7 billion monthly active users, $74 \%$ of them visit the platform on a daily basis, with 4.75 billion pieces of content shared daily and 510,000 comments posted every 60 seconds [23].

The potential capability of these platforms in exchanging information in the form of opinions, sentiments, and emotions, makes them an ideal place for consuming and spreading negative and extremist beliefs that promote terrorist activities for the achievement of political, economic, religious, or social goals [16]. For instance, the stunning mass-shootings in Christchurch were planned to get huge attention, leveraging Facebook, YouTube, Twitter, and Instagram platforms to make sure that a lot of people would hear about the deaths and the hate underpinning them. Official authorities have reported that before the attack, a 
Tweeter account was used to post a racist message in which the attackers identified the mosques that were later attacked [19]. Other criminal activities that followed these tactics include the Mumbai terrorist attack in 29 November 2008, Virginia shooting in 25 august 2015, the Brussels airport bombing in 22 Mar 2016, Manchester Arena bombing in 22 May 2017 and London Bridge attack in 3 Jun 2017. In all these cases, attackers used social media to spread their radical thoughts and criminal activities, before the attacks. A study conducted in [22], affirm that nearly 1 million accounts that promote terrorism and spread extremist thoughts were identified on Tweeter, in the last two years. Further, it is estimated there are over than 100,000 tweets of hate, racism and ethnic generated daily on Twitter [27]. This new reality means that governments, police forces and others involved in public safety, should paying attention to social media valuable content to track these criminals activities, and determine how to react effectively in the near term before they become a real problem [29]. Previous studies have argued that these platforms have the hidden potential to reveal valuable insights when analysis techniques are applied to their unstructured data. In this context, Sentiment Analysis (SA) or Opinion Mining (OM) [20] is one of the key emerging technologies that help navigating the large volume of sentiment rich data generated by users on social networking websites. SA is defined as a process that automates extracting of opinions, attitudes and emotions from a piece of text, through Natural Language Processing (NLP) methods $[12,20]$. It involves detecting whether the text expresses a positive, a negative, or a neutral opinion toward an entity (e.g., individual, organisation, event, topics, etc.) [17]. Unlike traditional data mining methods, sentiment analysis deal with unstructured data which is usually textual and messy such as documents, emails, user posts and comments on social media [24]. In the last years, Sentiment Analysis techniques have been intensively exploited to identify users interests or behaviours through the information extracted from social media, especially towards the distorted beliefs and negative sentiments $[5,11,25,30]$. Most of the proposed approaches in this area showed promising results and high accuracy in classifying negative users sentiments and opinions. However, most of these studies deal with English texts, while other languages, especially Arabic have received less attention. Despite being one of the fastest-growing languages in term of users on social media, the field of Arabic NLP is however not mature compared to English and other languages, for many reasons including the language complexity, variety of related dialects, an insufficient number of research publications and datasets gathered and analysed for such purpose [6]. Therefore, effective and more accurate Arabic sentiment analysis becomes the fundamental demand for analysing the huge amount of Arabic data available on social media and detect suspicious users.

This paper aims to bridge the gap in research concerning Arabic sentiment analysis, which can lead to a better understanding of the driving changes in different Arab countries and their impact on a global scale. To this end, we build a lexicon that contains positive and negative tokens (words). For the purpose of this study, a corpus of $500 \mathrm{mix}$ feelings tweets is collected. The collected data was manually selected in regard to the Saudi-Qatari conflict [26]. The conflict led to 
cutting relation between the two countries until the writing of this paper. Most of the conflict was waged through social media platforms, especially Twitter, where a huge number of Arabic tweets that spread hate and negative thoughts were pushed by armies of fake accounts known as "bots" to manipulate the public opinion, according to a BBC Arabic investigation [26]. The hate speech in these tweets created a strong tension between people in the media in those two countries. The main contribution of this paper is to understand to what extend the existing publicly available systems and algorithms are able to classify

a given Arabic tweet polarity. In addition, a more comprehensive lexicon of Arabic tokens is developed and evaluated based on manual annotation of the tweets from 6 native Arabic speakers. Then results were compared with other well-known systems and algorithms in order to demonstrate the efficacy of our lexicon.

The rest of the paper is organised as follows. Section 2 presents a review of current state-of-the-art literature on opinion mining and sentiment analysis of Twitter data. Section 3 describes the process of collecting the corpora from Twitter platform. Then, the generated mixed lexicon and the linguistic analysis of the obtained corpora are presented in section 4 . Section 5 presents the experimental results of the study and the findings are discussed in section 6 . Finally, section 7 reviews the content of the paper, presents the conclusions and outlines the potential work in the future.

\section{Related work}

In recent years, sentiment analysis becomes one of the fastest-growing research areas. In this context, many studies have been applied to effectively improve the understanding of user opinion on diverse challenging issues. For instance, Authors in [25] used the sentiments expressed by the Turkish people in their tweets to understand the public opinions towards the Syrian refugee crisis. Similarly, in [11], authors exploited the sentiment analysis of Twitter data to provide graphical visualisations about potential terrorism scenarios. The study demonstrated an example that social media and sentiment analysis technologies can play a critical role in effective response to terrorism physical activities by analysing shared information. In another recent work [5], authors proposed a sentiment analysis approach to classify the user-generated posts on Twitter as extremist or non-extremist. This approach achieved $90 \%$ of accuracy in the classification of the users sentiments. In [30], authors focused on studying the influence of hate speech on the behaviour of tweeter users. They reached $78.4 \%$ accuracy in detecting whether a tweet is hateful or not.

With regard to the Arabic language, there is a lack of research that addresses the sentiment analysis in this language, especially for detecting and classifying distorted beliefs and extremist sentiments [25, 30]. The proposed studies in this area used two main approaches for sentiment analysis; Machine learning and lexicon-based approach. The Lexicon-based Approach is an unsupervised method that relies on a sentiment lexicon [17], which is a lexicon of known and 
pre-compiled sentiment terms, phrases and even idioms. It matches the words in the lexicon with the data to determine the polarity [17, 25]. In this approach, sentiment scores are assigned to the opinion words describing how Positive, Negative and neutral the words contained in the lexicon are $[6,17]$. Lexicon-based approaches can be classified into lexicon-based approaches [17] and corpus-based approaches [20]. The later relies on human experts to annotate a set of data that can be used to train a classification model. The trained model can then be used to classify any new data item [17]. While machine learning techniques like Naive Bayes (NB), Maximum Entropy (ME), and Support Vector Machines (SVM) are usually used to classify the data into different classes $[6,17,20]$.

The first interesting method for sentiment analysis of Arabic documents was proposed in [2]. In this study, authors used a hybrid method for sentiment analysis. First, a lexicon-based approach is used to classify some documents, which will be used as a training dataset for ME method, which subsequently classifies some other documents. Then, the k-nearest model is used to classify the rest of the documents. To validate this approach, authors used a dataset that was collected from 1,143 posts which contain 8,793 opinions expressed in Arabic from three different domains: "education", "politics" and "sports". Authors reported an accuracy equal to $80.29 \%$ on detecting negative and positive statements. In one of the few attempts of Arabic extremist SA, Authors considered a binary (positive or negative) SA approach of English and Arabic hate/extremist web forum posts [1]. This approach focused on feature selection by using a wide array of English and Arabic stylistic attributes, including lexical, structural, and function-word style markers. For efficient feature selection for each sentiment class, they also developed an Entropy Weighted Genetic Algorithm (EWGA). The effectiveness of this approach was evaluated on two small datasets, each consisting of 1,000 posts written in English or Arabic. This approach achieved accuracy over $91 \%$ on both datasets. The main drawback of this approach was the extreme lack of pre-processing which is really crucial for Arabic text.

Given the cultural and linguistic differences across Arab world, inducing variations in semantics, some studies focused on developing SA for the different Arabic dialects used in the social media texts $[9,10,14]$. According to [31] there are four dominant dialects in the Arab world: Egyptian, Levantine, Gulf, Iraqi and Maghrebi. In this context, authors in [10] introduced a sentiment model for the Levantine dialect (ArSenTD-LEV) ${ }^{3}$. The proposed model used a corpus composed of 4,000 tweets retrieved from Levantine countries (Jordan, Lebanon, Palestine and Syria). For each tweet, the corpus specifies its overall sentiment and topic, the target to which the sentiment was expressed and how it is expressed (explicitly or implicitly). The experimental results confirmed the relevance of these annotations at improving the accuracy of SA classifiers. Other works in this direction proposed a lexicon-based technique to deal with dialectal Arabic [9]. In this approach, the authors used an online game ${ }^{4}$ that enables users to annotate large corpuses of text in a fun manner. Whereas, for the text analysis

\footnotetext{
3 The ArSenTD-LEV corpus is available atwww.oma-project.com

${ }^{4}$ http://kalimat.afnan.ws/
} 
and classification, they used the sentimental tag patterns and the sentimental majority approach. Authors reported $60.50 \%$ of accuracy for the sentimental majority approach while the sentimental tag patterns yielded lower accuracy of $60.32 \%$. In [14], authors discussed in details the challenges faced by SA of dialectal Arabic on social media, especially the Egyptian dialect. Later they proposed a method for automatically constructing sentiment lexicon for Egyptian dialect [15].

Some other studies focused on the scarcity of available datasets by providing new resources to support research advances in Arabic SA [20 - 23]. In [28], authors introduced the Opinion Corpus for Arabic (OCA), one of the earliest public Arabic corpus for SA. The dataset contains 500 movie reviews collected from different web pages and blogs in Arabic, 250 of them considered as positive reviews, and the other 250 as negative opinions. While AWATIF [3] was the first corpus for Arabic SA that employed both regular and crowd-sourcing annotation techniques. AWATIF contains 5342 sentences taken from 30 Wikipedia talk pages, Twitter and 2532 threaded conversations taken from seven Arabic forums. Later, the authors proposed SANA [4], a large-scale, multi-domain, multi-dialect, and multi-genre lexicon for sentiment analysis of the Arabic language and dialects. The lexicon automatically extends two manually collected lexicons HUDA (4,905 entries extracted from chat records in the Egyptian dialect) and SIFFAT (3,325 Arabic adjectives). In [21], authors prepared a sentiment analysis dataset gathered from Arabic tweets, called Arabic Sentiment Tweets Dataset (ASTD). ASTD consists of 10,000 tweets which are classified as objective, subjective positive, subjective negative, and subjective mixed. The authors constructed a seed sentiment lexicon from the dataset. In [8] authors presented an Arabic lexiconbased tool called Arabic Opinions Polarity Identification (AOPI). This tool relies on domain-specific lexicon approach for extracting opinions in posts written in Modern Standard Arabic (MSA) and dialectal Arabic. They compared it with SocialMention ${ }^{5}$ and SentiStrength ${ }^{6}$. Their results showed that AOPI is more accurate than the other tools. Studies in $[7,18]$ showed that most of SA tools are inefficient for extracting opinions in reviews written in MSA or in dialectal Arabic.

From the state of the art, it can be concluded that despite the speedy growth in the volume of Arabic opinionated posts on social media, Research in the area of Arabic SA is progressing at a very slow pace compared to that being carried out in English and other languages. Moreover, most of the available resources in this area are either of limited size, domain-specific or not publicly available. In addition, most of them had issues in terms of the quality of its content and annotation, which limiting advancement in Arabic sentiment analysis. Also, there is no study that compares publicly available sentiment analysis algorithms on limited Arabic text (tweets) with Lexicon and human opinions of both male and females.

\footnotetext{
${ }^{5}$ SocialMention: http://www.socialmention.com/

${ }^{6}$ SentiStrength: http://sentistrength.wlv.ac.uk/
} 


\section{Corpus Collection}

The corpus samples were collected manually by three different people from Twitter platform. There was no specific selection criteria followed, instead, the collectors were asked to browse Twitter platform naturally using a pre-setup account in which they tag any tweet that they think it relates to the matter (Saudi-Qatari politic conflict). In total, 500 tweets were collected during the period from 1 to 8 January 2019. Table 1 provides some statistical information about the collected corpus.

Table 1. Corpus Statistics

\begin{tabular}{|l|l|l|l|l|l|l|l|}
\hline \multirow{2}{*}{ Item } & \multicolumn{7}{|c|}{ Corpus overall stats } \\
\cline { 2 - 8 } & mean & std & min & $25 \%$ & $50 \%$ & $75 \%$ & $\max$ \\
\hline token & 21.30 & 10.47 & 1 & 16 & 20 & 24 & 55 \\
Char & 139.48 & 63.18 & 3 & 110 & 124 & 145 & 305 \\
Special char & 0.22 & 0.88 & 0 & 0 & 0 & 0 & 13 \\
link & 0.44 & 0.54 & 0 & 0 & 0 & 1 & 2 \\
hashtag & 1.65 & 1.59 & 0 & 1 & 1 & 2 & 10 \\
emojis & 0.23 & 0.76 & 0 & 0 & 0 & 0 & 7 \\
@ & 0.18 & 0.55 & 0 & 0 & 0 & 0 & 3 \\
\hline
\end{tabular}

The table shows the mean (average), standard deviation, min, max and quartiles of the listed items for the corpus tweets. The token expresses any single word, term, or symbol exist in a tweet that is separated by white space. However, the definition of the token can be controversy. In this paper context, the above definition is what we followed. For example; the tweet below has five tokens.

$$
\text { "\# محمد بن سلمان طموحنا عنان السماء SA" }
$$

1. \#محمد بن سلمان: means person: Mohammed bin Salman

2. طموحنا: means: noun: Our ambition

3. عنان: means: noun: highest

4. السماء: means: noun: sky

5. SA: means acronym: Saudi Arabia

The given translation is not very accurate as it expressed word by word not as a whole sentence translation in which the English meaning could be not very clear. From the table, it can be seen that the average tweet has around 21 words. The char indicates any single character in a tweet, for instance, the word طموحن has the following characters: allows by max 280 characters per tweet which used to be 140 characters. The 
averaged tweet in the collected corpus has around 139 characters. The special char expresses exclamation (!) and question marks (?). The existing of such marks in a tweet could express a type of feeling. For example, the exclamation mark in a short sentence could express very strong feeling. While the existing of question mark could indicate that the tweet is kind of a question in a way that the author is not agreeing with context or the point that is made by someone else. From the table, the existing of these marks in the corpus tweet is around 0.22 per tweet. Which means that one in every five tweets could include such mark. Finally, "@" indicates the existence of "@" in the corpus tweets whether it belongs to a username or email or any type of context. Table 2 lists the most common tokens in the collected corpus.

Table 2. Most Frequent Tokens

\begin{tabular}{|c|c|c|c|c|c|}
\hline Token & \begin{tabular}{|l|} 
English \\
translation
\end{tabular} & Frequency & Token & \begin{tabular}{|l|} 
English \\
translation
\end{tabular} & Frequency \\
\hline في & In & 245 & من & From & 212 \\
\hline على & On & 117 & | محمد بن سلمان \# & That & 73 \\
\hline ولي العهد \#\# & crown prince & 71 & |السعودية \# | & Saudia & 65 \\
\hline أن & That & 63 & بن & Son & 62 \\
\hline 9 & And & 60 & مع & With & 59 \\
\hline سلمان & Salman & 56 & & & \\
\hline
\end{tabular}

It can be seen that most of the listed word are stop words and name of the crown prince of Saudi Arabia as well as the king of the country.

\section{Lexicon Generation}

In this study a mixed lexicon was used since the current literature review did not provide a suitable Arabic lexicon that could fulfill the aims of this study. The lexicon created was a combination of "AraSentiLexicon V 1.0" made by Nora Al-Tweiresh in 2016, along with an Arabic translation of Bing Lius Lexicon. The translated Bing Liu lexicon has been manually edited and numerous sentiment words added in order to includes all cases of sentiments in Arabic language for male, female, past, present, future, formal, informal as well as other cases that can cause differences in the Arabic language. At the end, the positive lexicon produces was consisted of more than 61,600 positive words and the negative lexicon consisted of more than 77,900 negative words.

Both lexicons can be downloaded from the link below ${ }^{7}$. Table 3 lists the most common usernames in the corpus from which the tweets were collected. Any user who has four or more tweets is included in the figure along with their number of

\footnotetext{
7 Abduallah Arabic Lexicon: http://shiaeles.net/datasets/Arabic_Lexicon_Abduallah.7z
} 
tweets. Those 22 users (out of 202) form $44 \%$ of the total percentage of tweets in the corpus. While 157 tweets out of these 500 tweets (total corpus samples) belong to only the top seven accounts most of which are news accounts.

Table 3. Most Accounts in the Corpus

\begin{tabular}{|l|c|l|c|}
\hline Screen name & Count of Tweets & Screen name & Count of Tweets \\
\hline @AJABreaking & 30 & @TurkiShalhoub & 6 \\
@mshinqiti & 30 & @m3takl & 6 \\
@Benguennak & 29 & @AjelNews24 & 5 \\
@hureyaksa & 25 & @spagov & 5 \\
@ELHAMBADER1 & 15 & @AlkhaleejOnline & 4 \\
@DrMahmoudRefaat & 8 & @HashKSA & 4 \\
@AJArabic & 7 & @MALHACHIMI 4 & 4 \\
@GamalSultan1 & 7 & @MBNsaudi & 4 \\
@Saudi_24 & 7 & @aa_arabic & 4 \\
@Raed_Fakih & 6 & @jamalrayyan & 4 \\
@SaudiNews50 & 6 & @saudibus222 & 4 \\
\hline
\end{tabular}

\section{Experimental analysis}

In order to measure the performance of the examined approaches, two aspect should be considered, word aspect and general overview aspect. The word aspect is when the matching is based on the words within a tweet with the lexicon and accuracy can it find the negative and positive words within a tweet, this also includes how comprehensive are the positive and negative lexicon. The other aspect is the general polarity aspect is how accurately can the method/software understand the general sentiment of the tweet and be able to classify it accurately. The corpus tweets were classified and annotated into one of three classes, these are; positive, negative or neutral. This is done by requiting 6 adult people ( 3 males and 3 females) to perform a manual annotation. Also, the polarity of the tweets were classified by the developed lexicon that this study used. Table 4 presents the overall corpus sentiment polarity. It can be seen that, the developed lexicon positive sentiment rate is close to the human based rate. While the negative and neutral sentiment is different.

In comparison with publicly available sentiment analysis algorithms, these include SentiStrength, uClassify and Vader, the developed Arabic dictionary (lexicon) is the most closest to the Human result as illustrated in Fig. 1. However, this is also shows that the problem of analyzing Arabic sentiment is not an easy tasks. As the results significantly varies among the tested approaches. Moreover, it is not only varies among the examined algorithms, but also among those requited human annotators. As illustrated in Figure 2, it can be seen that around $75 \%$ percentage of the corpus has an agreements of 4 or more annotators. While the remainder are agreed with three or less people. This reviles how even human 
Table 4. Corpus Sentiment Polarity

\begin{tabular}{|l|l|l|}
\hline Sentiment & \multicolumn{2}{|l|}{ Methode } \\
\cline { 2 - 3 } & Lexicon & Human \\
\hline Positive & $43 \%$ & $41 \%$ \\
Negative & $33 \%$ & $14 \%$ \\
Netrutal & $25 \%$ & $45 \%$ \\
Total points & 1,330 & N/A \\
\hline
\end{tabular}

themselves have different views and feelings on the same given text. In which, developing a sentiment analysis system is not a simple task.

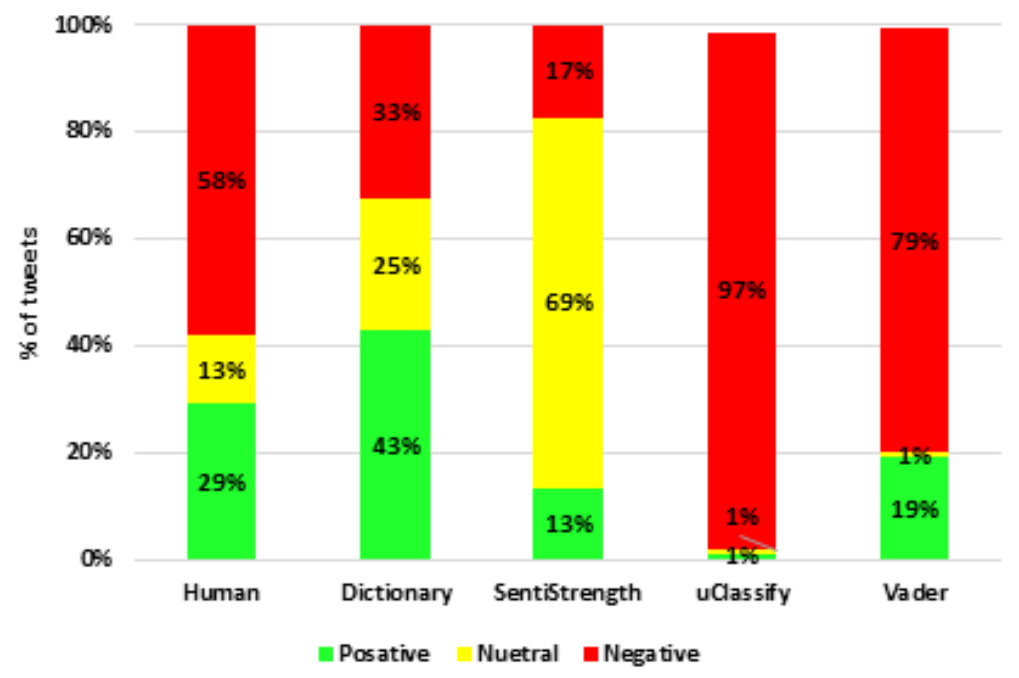

Fig. 1. Overall Corpus sentiment polarity

To fairly evaluate and compare those selected approaches and systems with human annotations, we filtered the corpus to have only those tweets where there is an agreement among the human annotators of 4 and more. This resulted in reduction on the data to 121 tweets (out of 500). Table 5 presents the agreements as percentage and number of those tweets for each approach and the developed lexicon along with those 4 human agreement. 


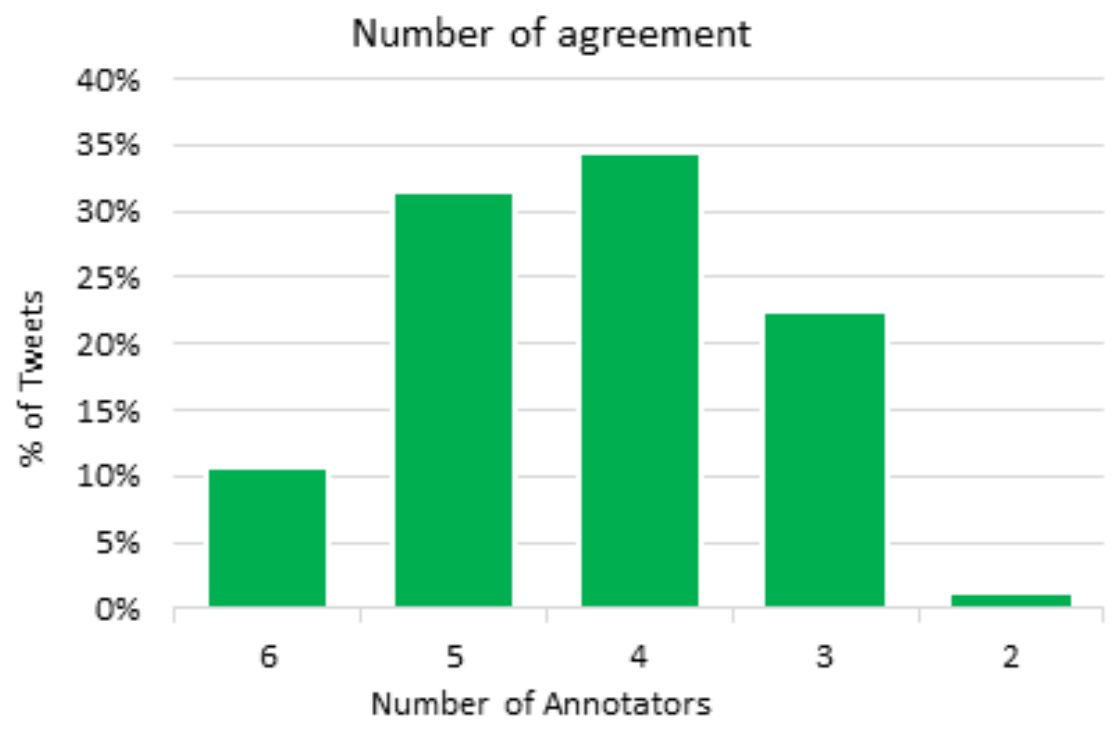

Fig. 2. Overall Corpus Sentiment Polarity

Table 5. Agreement of tools with human annotations

\begin{tabular}{|l|l|l|l|l|}
\hline \multirow{2}{*}{ Sentiment } & \multicolumn{4}{|c|}{ Method } \\
\cline { 2 - 5 } & Lexicon & SentiStrength & uClassify & Vader \\
\hline Positive & $83 \%(29)$ & $23 \%(8)$ & $0 \%(0)$ & $1 \%(1)$ \\
Negative & $57 \%(40)$ & $24 \%(17)$ & $94 \%(66)$ & $71 \%(49)$ \\
Natural & $20 \%(4)$ & $75 \%(12)$ & $0 \%(0)$ & $6 \%(1)$ \\
Overall & $60 \%(70)$ & $30 \%(37)$ & $54 \%(66)$ & $42 \%(51)$ \\
\hline
\end{tabular}




\section{Discussion}

According to the presented results of the analysis, it can be stated, that the lexicon performance is better than the other methods. However, is noteworthy that sometimes the results were not correct due to some missing words from our used lexicons. Another reason is that a tweet carries mixed opinions and sentiment which can cause faults. Further reason that can lead to less accurate results is the nature of the Arabic language itself, as there is several vocals such as نَ نَ، نُ، ن and many other vocals; in this example, the letter ن is called Noon which similar to the letter $\mathrm{N}$ in English. However, the letter was written with different vocals in the example which will make the sound different in each case, in the first case where the letter was $\dot{ن}$, the letter sounds $\mathrm{Na}$, in the second case the letter $\stackrel{ن}{ن}$ sounds like No, and in the last case of the example the letter sound like Ni. There are more vocals in Arabic language and these vocals can apply to Arabic letters in every word and sometimes it can be the same word with different vocals, and hence it can refer to different meanings. An example of this the word سَحْْْ and the first sound like Silm which means peace, the second word sounds like Sollum which means a ladder, now both of the words have exactly the same letters with just different vocals. The issue is in about $95 \%$ from the Arabic posts on the internet, as Arabs do not usually use the vocals because it is easy for them to know the intended word by the context, but when it comes to the computer, it is an issue to understand the exact meaning the user refers to.

\section{Conclusion}

This research dealt with the significant problem of the lack of comprehensive Arabic lexicon. The results of the analysis of the 500 Arabic tweets in respect to the sentiments and the opinions of Twitter active users regarding the examined subject showed that there are some agreements between the developed lexicon and the human annotators. However, the results are still around $75 \%$ accurate comparing the human annotators, therefore, there is a strong need to investigate other advanced approaches such as machine learning and deep learning based methods along with the lexicon, being able to capture the latent meaning and feelings of the tweets.

\section{ACKNOWLEDGEMENT}

The authors of this paper would like to thank Salam Ketab, Hussam Mohammed, Mona Almana, Aysar Hasan and Yasameen Mahih for the time devoted in annotating the tweets manually. 


\section{Bibliography}

[1] Abbasi, A., Chen, H., Salem, A.: Sentiment analysis in multiple languages: Feature selection for opinion classification in web forums. ACM Transactions on Information Systems (TOIS) 26(3), 12 (2008)

[2] Abdul-Mageed, M., Kuebler, S., Diab, M.: Samar: a system for subjectivity and sentiment analysis of arabic social media (2012)

[3] Abdul-Mageed, M., Diab, M.T.: Awatif: A multi-genre corpus for modern standard arabic subjectivity and sentiment analysis. In: LREC. vol. 515, pp. 3907-3914. Citeseer (2012)

[4] Abdul-Mageed, M., Diab, M.T.: Sana: A large scale multi-genre, multidialect lexicon for arabic subjectivity and sentiment analysis. In: LREC. pp. 1162-1169 (2014)

[5] Ahmad, S., Asghar, M.Z., Alotaibi, F.M., Awan, I.: Detection and classification of social media-based extremist affiliations using sentiment analysis techniques. Human-centric Computing and Information Sciences 9(1), 24 (2019)

[6] Al-Ayyoub, M., Khamaiseh, A.A., Jararweh, Y., Al-Kabi, M.N.: A comprehensive survey of arabic sentiment analysis. Information Processing \& Management 56(2), 320-342 (2019)

[7] Al-Kabi, M., Al-Qudah, N.M., Alsmadi, I., Dabour, M., Wahsheh, H.: Arabic/english sentiment analysis: an empirical study. In: The Fourth International Conference on Information and Communication Systems (ICICS 2013). pp. 23-25 (2013)

[8] Al-Kabi, M., Alsmadi, I., Khasawneh, R.T., Wahsheh, H.: Evaluating social context in arabic opinion mining. Int. Arab J. Inf. Technol. 15(6), 974-982 (2018)

[9] Al-Subaihin, A.S., Al-Khalifa, H.S.: A system for sentiment analysis of colloquial arabic using human computation. The Scientific World Journal 2014 (2014)

[10] Baly, R., Khaddaj, A., Hajj, H., El-Hajj, W., Shaban, K.B.: Arsentd-lev: A multi-topic corpus for target-based sentiment analysis in arabic levantine tweets. arXiv preprint arXiv:1906.01830 (2019)

[11] Cheong, M., Lee, V.C.: A microblogging-based approach to terrorism informatics: Exploration and chronicling civilian sentiment and response to terrorism events via twitter. Information Systems Frontiers 13(1), 45-59 (2011)

[12] Danneman, N., Heimann, R.: Social media mining with R. Packt Publishing Ltd (2014)

[13] Dorsey, J.: Twitter by the numbers: Stats, demographics fun facts, [online] OMNICORE. Available: url = https://www.omnicoreagency.com/twitterstatistics/, [Accessed 1 September 2019] 
[14] El-Beltagy, S.R., Ali, A.: Open issues in the sentiment analysis of arabic social media: A case study. In: 2013 9th International Conference on Innovations in Information Technology (IIT). pp. 215-220. IEEE (2013)

[15] ElSahar, H., El-Beltagy, S.R.: A fully automated approach for arabic slang lexicon extraction from microblogs. In: International conference on intelligent text processing and computational linguistics. pp. 79-91. Springer (2014)

[16] Hossain, M.S.: Social media and terrorism: Threats and challenges to the modern era. South Asian Survey 22(2), 136-155 (2015)

[17] Kharde, V., Sonawane, P., et al.: Sentiment analysis of twitter data: a survey of techniques. arXiv preprint arXiv:1601.06971 (2016)

[18] Khasawneh, R.T., Wahsheh, H.A., Al-Kabi, M.N., Alsmadi, I.M.: Sentiment analysis of arabic social media content: a comparative study. In: 8th International Conference for Internet Technology and Secured Transactions (ICITST-2013). pp. 101-106. IEEE (2013)

[19] Lopatto, E.: The mass shooting in new zealand was designed to spread on social media, [online] The Virage. Available: url = https://www.theverge.com/2019/3/15/18266859/new-zealand-shootingvideo-social-media-manipulation, [Accessed 1 September 2019]

[20] Medhat, W., Hassan, A., Korashy, H.: Sentiment analysis algorithms and applications: A survey. Ain Shams engineering journal 5(4), 1093-1113 (2014)

[21] Nabil, M., Aly, M., Atiya, A.: Astd: Arabic sentiment tweets dataset. In: Proceedings of the 2015 Conference on Empirical Methods in Natural Language Processing. pp. 2515-2519 (2015)

[22] News, B.: Twitter suspends almost a million accounts for promoting terrorism in two years, [online] Breaking News. Available: $\operatorname{url}=$ https://www.breakingnews.ie/tech/twitter-suspends-almosta-million-accounts-for-promoting-terrorism-in-two-years-806566.html, [Accessed 1 September 2019]

[23] Noyes, D.: The top 20 valuable facebook statistics updated september 2019, [online] ZEPHORIA. Available: url = https://zephoria.com/top-15valuable-facebook-statistics/, [Accessed 1 September 2019]

[24] Oza, K.S., Naik, P.G.: Prediction of online lectures popularity: a text mining approach. Procedia Computer Science 92, 468-474 (2016)

[25] Öztürk, N., Ayvaz, S.: Sentiment analysis on twitter: A text mining approach to the syrian refugee crisis. Telematics and Informatics 35(1), 136147 (2018)

[26] Pinnell, O.: The online war between qatar and saudi arabia, [online] BBC Arabic News. Available: url = https://www.bbc.co.uk/news/blogstrending-44294826, [Accessed 1 September 2019]

[27] Relia, K., Li, Z., Cook, S.H., Chunara, R.: Race, ethnicity and national origin-based discrimination in social media and hate crimes across 100 us cities. In: Proceedings of the International AAAI Conference on Web and Social Media. vol. 13, pp. 417-427 (2019) 
[28] Rushdi-Saleh, M., Martín-Valdivia, M.T., Ureña-López, L.A., Perea-Ortega, J.M.: Oca: Opinion corpus for arabic. Journal of the American Society for Information Science and Technology 62(10), 2045-2054 (2011)

[29] Stanton, J.: Examining the Use of Social Media by United States Senators. Ph.D. thesis, Information and Design TechnologyState University of New York (2014)

[30] Watanabe, H., Bouazizi, M., Ohtsuki, T.: Hate speech on twitter: A pragmatic approach to collect hateful and offensive expressions and perform hate speech detection. IEEE Access 6, 13825-13835 (2018)

[31] Zaidan, O.F., Callison-Burch, C.: Arabic dialect identification. Computational Linguistics 40(1), 171-202 (2014) 